\title{
BMJ Open Associations between pentraxin 3 and severity of coronary artery disease
}

\author{
Hua Liu, Shaofeng Guan, Weiyi Fang, Fang Yuan, Min Zhang, Xinkai Qu
}

To cite: Liu H, Guan S, Fang W, et al. Associations between pentraxin 3 and severity of coronary artery disease. BMJ Open 2015;5: e007123. doi:10.1136/ bmjopen-2014-007123

- Prepublication history for this paper is available online. To view these files please visit the journal online (http://dx.doi.org/10.1136/ bmjopen-2014-007123).

$\mathrm{HL}$ and SG contributed equally.

Received 7 November 2014 Revised 4 March 2015 Accepted 5 March 2015

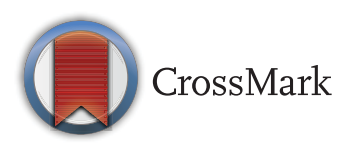

Department of Cardiology, Shanghai Chest Hospital, Affiliated to Shanghai Jiao Tong University, Shanghai, China

Correspondence to Dr Xinkai Qu; qxkmedsci@163.com

\section{ABSTRACT}

Objective: To investigate the associations between plasma levels of pentraxins 3 (PTX3) and $C$ reactive protein (CRP) and the severity of coronary artery lesions.

Design and methods: 60 patients with coronary heart disease $(\mathrm{CHD})$ who underwent coronary angiography (CAG) in our hospital were included. Plasma was collected during CAG. The coronary Gensini score was used to evaluate the severity of coronary artery lesions. Associations between Gensini scores and plasma levels of PTX3 and CRP were analysed. Patients with estimated glomerular filtration rate $<60 \mathrm{~mL} / \mathrm{min} / 1.73 \mathrm{~m}^{2}$ were included in the chronic renal dysfunction subgroup.

Results: A linear correlation was observed between PTX3 and the Gensini score $(r=0.513, p<0.001)$. Oneway analysis of variance showed that PTX3 levels were significantly higher in patients with Gensini scores $>90$ compared with patients with scores of 46-90 or $<45$ (0-45:4.8 $\pm 0.8,46-90: 6.7 \pm 1.2,>90: 7.7 \pm 2.0, p<0.001)$. Stepwise multiple linear regression showed that PTX3 levels were significantly associated with Gensini score in patients with chronic renal dysfunction ( $p=0.012)$, while no significant association was found for CRP.

Conclusions: PTX3 levers were positively associated with the severity of coronary artery lesions. PTX 3 is closely associated with the severity of coronary artery stenosis in patients with chronic renal dysfunction.

\section{INTRODUCTION}

Inflammation plays a critical role in the development and progression of coronary heart disease (CHD), and increased local inflammation may induce plaque rupture, which has been acknowledged as the initial factor in acute coronary syndrome. ${ }^{1}$ Therefore, finding inflammatory factors that accurately reflect the instability of atherosclerotic plaques has been accepted as an important method for the early prediction of cardiovascular events. $^{2}$

Pentraxin 3 (PTX3) and $\mathrm{C}$ reactive protein (CRP) are two components of the pentraxin superfamily which are considered to be important inflammatory factors that play critical roles in the progression of atherosclerosis. $^{3-5}$ The predictive value of CRP for CHD has already been widely investigated. However,
Strengths and limitations of this study

- Cross-sectional study without follow-up.

- Small sample size.

CRP is produced in the liver and is induced by several non-cardiac diseases, so its specificity is still being debated. ${ }^{6}{ }^{7}$ In contrast, PTX3 is produced in the cardiac circulation, ${ }^{8}$ before CRP, in patients with acute coronary syndrome. ${ }^{9}$ Therefore, PTX3 could be superior to CRP as an inflammatory biomarker for CHD. $^{10}$

However, only a few studies have investigated the association between PTX3 or CRP levels and the severity of coronary artery lesions. Two studies in patients with angina have shown that there is a strong correlation between coronary sinus plasma PTX3 concentrations and severity of atherosclerosis. ${ }^{11}{ }^{12}$ There is a negative correlation with CT density of plaques, which is not seen with CRP. ${ }^{11}$ Recent studies found that PTX3 is also expressed in renal tissues ${ }^{13}$ and PTX3 is an independent determinant of the severity of coronary artery disease (CAD) in patients with stage 2 and 3 kidney disease. ${ }^{14}$ So more studies are needed to fully evaluate this relationship in different populations of patients, especially the severity of coronary artery stenosis in patients with renal dysfunction. In the present study, the coronary Gensini score was used to evaluate the severity of coronary artery stenosis in Chinese patients with CHD susceptibility. We undertook a small-scale preliminary study in order to test the value of this method of investigation. The association between the Gensini score and PTX3 or CRP levels was analysed to explore the correlation between the pentraxin superfamily and CHD, especially the correlation with the severity of coronary artery stenosis in patients with chronic kidney dysfunction (CKD). This information will then be used as a basis for larger scale studies involving a detailed investigation of PTX3 in Chinese patients with CAD and CKD. 


\section{MATERIALS AND METHODS}

\section{Patients}

This was a study in consecutive patients with CHD susceptibility who underwent coronary angiography (CAG) and stenting at the Shanghai Chest Hospital affiliated to Shanghai JiaoTong University between October 2013 and November 2013. During this short inclusion period, all eligible patients with stable coronary artery diseases agreed to participate. Patients with one or more of the following features were excluded: (1) complicated with infectious diseases on admission; (2) immune system disease and/or on glucocorticoid therapy; (3) malignant tumour; (4) clinical or CAG data unavailable; and (5) CKD from another cause than CHD, such as patients with nephritis, but not those with diabetes or hypertension; (6) valvular heart disease; (7) had suffered heart failure including previous myocardial infarction (MI) or had undergone percutaneous coronary intervention (PCI); (8) acute coronary syndromes.

Renal dysfunction was diagnosed as patients with a symptom of renal dysfunction and/or a reduced glomerular filtration rate (GFR) $\left(<60 \mathrm{~mL} / \mathrm{min} / 1.73 \mathrm{~m}^{2}\right)$ for more than 3 months. Hypertension was defined as systolic blood pressure $>140 \mathrm{~mm} \mathrm{Hg}$ or diastolic blood pressure $>90 \mathrm{~mm} \mathrm{Hg}$ on admission, or taking antihypertensive drugs. Diabetes was defined as fasting blood-glucose $\geq 7.9 \mathrm{mmol} / \mathrm{L}$, or $2 \mathrm{~h}$ postprandial blood glucose $\geq 11.1 \mathrm{mmol} / \mathrm{L}$, or taking hypoglycaemic drugs.

The study protocol was approved by the ethics committee of our hospital, and all participants provided written informed consent.

\section{Procedures of CAG}

Patients were placed in the supine position and received local anaesthesia with $5-10 \mathrm{~mL}$ of lidocaine $(2 \%)$. The femoral or radial artery was selected for vascular access. The Seldinger technique was performed using a bevelled, hollow needle. A 6 Fr artery sheath catheter was implanted and $2000 \mathrm{IU}$ of unfractionated heparin was injected into the artery. Judkin JL 3.5 (or 4.0) and JL 4.0 angiographic catheters were implanted at the left and right coronaria dextra with the guidance of a 0.035 inch guide wire, and then contrast medium (Iohexol) was injected for CAG.

\section{Plasma collection and process}

During CAG, some arterial blood needed to be drawn when implanting the $6 \mathrm{Fr}$ or $7 \mathrm{Fr}$ artery sheath catheter to ensure the patency of the catheter, and this blood was collected for further examinations. After collection, the blood was mixed in a vacuum tube with precooled EDTA and aprotinin. Plasma was collected by centrifugation at $4^{\circ} \mathrm{C}, 3000 \mathrm{rpm}$ for $15 \mathrm{~min}$ and was stored at $-70^{\circ} \mathrm{C}$.

\section{Quantitative evaluation of the severity of coronary artery stenosis}

CAG was performed by experienced cardiologists. Coronary artery lesions were evaluated in the left anterior oblique, right anterior oblique and head-feet axes to assess stenosis severity of the right coronary artery, left main coronary artery, left anterior descending branch (LAD) and left circumflex (LCX) branch. The evaluation was performed according to the standard criterion for evaluating coronary artery stenosis recommended by the American Heart Association (AHA) ${ }^{15}{ }^{16}$ and by the same clinician to avoid subjective errors. According to the degree of stenosis, the stenosis of the coronary artery was classified as $\leq 25 \%, 50 \%, 75 \%, 90 \%, 99 \%$ or $100 \%$ (complete occlusion). The severity of each blood vessel stenosis was quantitatively evaluated by the Gensini scoring system. ${ }^{17}$ In brief, 0 indicates no abnormality, 1 indicates stenosis $\leq 25 \%, 2$ indicates stenosis $\leq 50 \%, 4$ indicates stenosis $\leq 75 \%, 8$ indicates stenosis $\leq 90 \%, 16$ indicates stenosis $\leq 99 \%$, and 32 indicates stenosis of $100 \%$. Evaluation of each coronary branch was also performed as the scores multiplied by 5 for the left main coronary artery, by 2.5 for the proximal LAD, by 1.5 for the middle LAD, by 1 for the distal LAD, by 1 for the first diagonal branch (DIAG), by 0.5 for the second DIAG, by 2.5 for the proximal LCX, by 1 for the distal LCX and posterior descending branch, and by 0.5 for the posterior branch; while for the right coronary artery it was performed as the scores multiplied by 1 for the proximal, middle and distal RAD and posterior descending branch, and by 0.5 for the posterior branch. The total score of each patient was calculated by adding the scores of each branch. The patients were then divided into three groups according to the total score $(0-45$, $46-90$ and $>90$ ).

\section{Glomerular filtration rate}

GFR was calculated according to the modified MDRD formula: estimated glomerular filtration rate (eGFR) $\left(\mathrm{mL} / \mathrm{min} / 1.73 \mathrm{~m}^{2}\right)=175 \times($ plasma creatinine $)-1.234 \times$ (age) $-0.179 \times($ female $\times 0.79)$. This was modified for a Chinese population according to a previous method. ${ }^{18}$

\section{Plasma PTX3 and CRP levels}

Serum levels of PTX3 and CRP were measured using ELISA kits. hPTX3/TSG-14 and human CRP Quantikine ELISA kits (R\&D Systems, China Co. Ltd., Shanghai, PRC).

\section{Statistical analysis}

Continuous data with a normal distribution are presented as means and SDs, while continuous data with a non-normal distribution are presented as medians and IQRs. Student $t$ test or $t$ test was used for the comparison of normally distributed continuous data with equal variances. The Wilcoxon rank-sum test was used for the analysis of continuous data with a non-normal distribution or with unequal variances. One-way analysis of variance was used for the analysis of continuous data with a normal distribution and equal variances between three or more groups, and the Kruskal-Wallis test was used for the comparison of continuous data with a non-normal 
Table 1 Characteristics of patients according to the Gensini score

\begin{tabular}{|c|c|c|c|c|}
\hline & \multicolumn{4}{|l|}{ Gensini score } \\
\hline & $0-45$ & $46-90$ & $>90$ & p Value \\
\hline Number (male) & $9(4)$ & $21(18)$ & $30(23)$ & \\
\hline Mean age (years) & $72.0 \pm 8.5$ & $70.1 \pm 10.6$ & $68.3 \pm 12.3$ & 0.70 \\
\hline Weight $(\mathrm{kg})$ & $67.1 \pm 6.3$ & $66.4 \pm 8.8$ & $64.3 \pm 9.8$ & 0.59 \\
\hline Hypertension & 7 & 15 & 16 & 0.26 \\
\hline Diabetes & 2 & 4 & 10 & 0.50 \\
\hline HDL-cholesterol (mmol/L) & $1.3 \pm 0.4$ & $1.4 \pm 0.4$ & $1.4 \pm 0.3$ & 0.86 \\
\hline LDL-cholesterol (mmol/L) & $2.9 \pm 0.4$ & $3.0 \pm 0.6$ & $2.8 \pm 0.6$ & 0.68 \\
\hline Creatinine $(\mu \mathrm{mol} / \mathrm{L})$ & $67.4 \pm 28.2$ & $79.9 \pm 36.0$ & $82.4 \pm 43.2$ & 0.60 \\
\hline eGFR $\left(\mathrm{mL} / \mathrm{min} / 1.73 \mathrm{~m}^{2}\right)$ & $84.7 \pm 27.0$ & $82.7 \pm 42.6$ & $81.5 \pm 38.0$ & 0.95 \\
\hline РТX3 $(\mu \mathrm{g} / \mathrm{L})$ & $4.8 \pm 0.8$ & $6.7 \pm 1.2$ & $7.7 \pm 2.0$ & $<0.001$ \\
\hline CRP (mg/L) & $4.0(2.0,7.5)$ & $8.0(3.0,15.0)$ & $12.5(8.75,19.0)$ & 0.002 \\
\hline
\end{tabular}

CRP, C reactive protein; eGFR, estimated glomerular filtration rate; HDL, high-density lipoprotein; LDL, low-density lipoprotein; PTX3,

pentraxins 3 .

distribution or with unequal variances among three or more groups. Pearson linear regression was used to analyse correlations. Stepwise multiple linear regression with an inclusion and exclusion level of 0.05 was used to identify factors associated with the Gensini score. All statistical analyses were performed using SPSS V.19.0 (IBM, Armonk, New York, USA). p Values $<0.05$ were considered statistically significant.

\section{RESULTS}

\section{Patient characteristics}

Table 1 presents patient characteristics according to the Gensini score. All three groups were comparable for gender, age, weight, hypertension, diabetes, high-density lipoprotein cholesterol, low-density lipoprotein cholesterol, creatinine and eGFR (all p>0.05). As the patients were recently diagnosed, they were not on any medication for treatment of their stable CAD during the study period.

\section{Association between PTX3, CRP and the Gensini score}

Increasing the Gensini score was associated with increasing levels of PTX3 (0-45:4.8 $\pm 0.8,46-90: 6.7 \pm 1.2$, > $90: 7.7$ $\pm 2.0, \mathrm{p}<0.001)$ and CRP $(0-45: 4.0(2.0-7.5), 46-90: 8.0$ (3.0-15.0), >90:12.5 (8.75-19.0), $\mathrm{p}=0.002)$ (table 1).

The PTX3 level was associated with the Gensini score of the patients $(\mathrm{r}=0.513, \mathrm{p}<0.001)$ (figure 1). The CRP level was weakly associated with the Gensini score of the patients ( $\mathrm{r}=0.261, \mathrm{p}=0.044)$ (figure 2$)$.

Stepwise multiple linear regression analysis showed that only PTX3 levels were independently associated with the Gensini score (table 2).

\section{Association between the PTX3 or CRP level and the Gensini score in patients with CKD}

Table 3 shows that all three Gensini score groups were comparable for all studied variables.

Stepwise multiple linear regression showed that only PTX3 was an independent predictor in patients with CKD $\left(\right.$ eGFR $\left.<60 \mathrm{~mL} / \mathrm{min} \cdot 1.73 \mathrm{~m}^{2}\right)($ table 4$)$.

\section{DISCUSSION}

The aim of this study was to investigate the associations between plasma levels of PTX3 and CRP and the severity of coronary artery lesions and also their particular

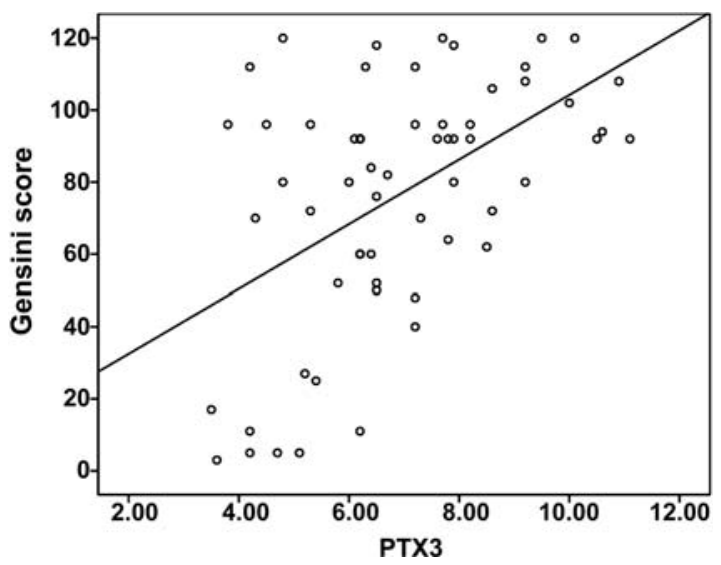

Figure 1 Correlation between the pentraxins 3 (PTX3) level and the Gensini score.

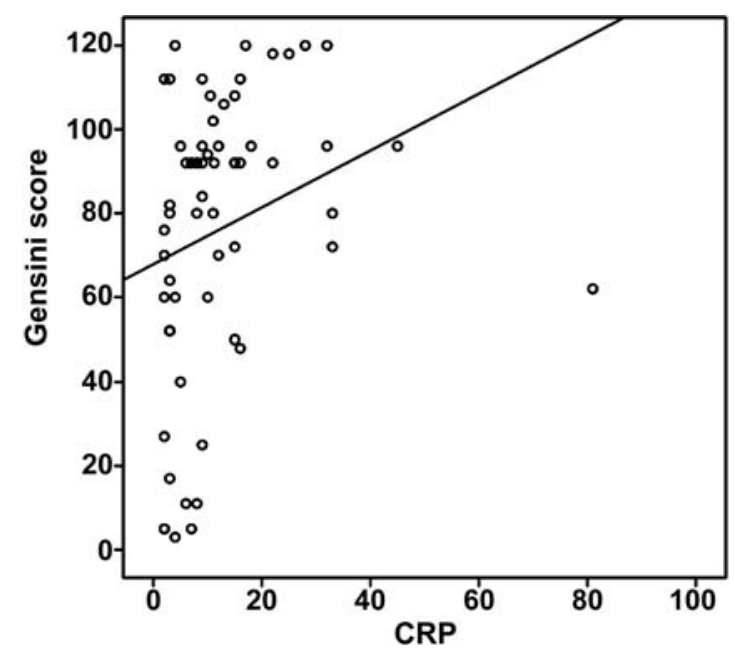

Figure 2 Correlation between the $\mathrm{C}$ reactive protein (CRP) level and the Gensini score. 
Table 2 Stepwise multiple linear regression of clinical characteristics and the Gensini score in all patients

\begin{tabular}{lrl}
\hline & \multicolumn{1}{l}{} & $\mathbf{p}$ Value \\
\hline Age & -0.174 & 0.863 \\
BMI & -1.776 & 0.082 \\
With complications & -0.202 & 0.380 \\
eGFR & 0.706 & 0.483 \\
PTX3 & 4.659 & 0.001 \\
CRP & 1.299 & 0.200
\end{tabular}

BMI, body mass index; CRP, C reactive protein; eGFR, estimated glomerular filtration rate; PTX3, pentraxins 3 .

associations in the subgroup of patients with renal dysfunction. Our results showed that there was a linear relationship between PTX3 and the Gensini score. PTX3 levels were significantly higher in patients with Gensini scores $>90$ compared with patients with scores $45-90$ or $<45$. PTX3 levels were also significantly associated with the Gensini score in patients with CKD; however, no significant association was found for CRP. These results suggest that PTX3 may be a valuable marker for severity of CAD.

To the best of our knowledge, this is the first study in a Chinese population to use the Gensini score system to evaluate the severity of CAD. However, other studies have also shown that PTX3 has the potential to be a more specific marker for the severity of CAD, as measured by the Gensini score, than CRP. ${ }^{11} 12{ }^{14}$ One of those studies concentrated on patients with stage 2 and 3 kidney disease. ${ }^{14}$ So our study is in agreement with the previous research. PTX3 is expressed in atherosclerotic plaques, mainly in macrophages and neutrophils, suggesting that PTX3 may be involved in the progression of atherosclerotic plaque. ${ }^{19}$ In one study, it was found that PTX3 may be an indicator of coronary plaque vulnerability. ${ }^{11}$ If this is the case, PTX3 has the potential to be a valuable tool not only in predicting severity of $\mathrm{CAD}$ but also in the prognosis of patients with CAD, as this is the first step in the process of acute coronary syndrome. ${ }^{1}$
Table 4 Stepwise multiple linear regression of clinical characteristics and the Gensini score in patients with renal dysfunction

\begin{tabular}{lrc}
\hline & \multicolumn{1}{c}{$\mathbf{r}$} & $\mathbf{p}$ Value \\
\hline Age & 1.193 & 0.251 \\
BMl & -1.129 & 0.277 \\
With complications & -0.291 & 0.775 \\
PTX3 & 2.665 & 0.018 \\
CRP & -0.216 & 0.832 \\
\hline BMI, body mass index; CRP, C reactive protein; PTX3, pentraxins 3.
\end{tabular}

In patients with acute coronary syndrome, chronic heart failure or those undergoing coronary stenting, plasma PTX3 levels may be higher than CRP levels, ${ }^{9}$ and thus could be used as an early biomarker for risk analysis. $^{20}$ PTX3 levels peak at about $7 \mathrm{~h}$ after acute MI, which is substantially earlier than CRP, and thus PTX3 could be a better independent predictor of CHD than $\mathrm{CRP}^{20}$ PTX3 could also be used as a predictor for restenosis following coronary artery bypass graft and circulatory failure. $^{21}$ For patients with ST-elevation MI (STEMI), a PTX3 >10.73 ng/mL could predict 3-month overall mortality after adjusting for other major risk factors including CRP. $^{22}$ In patients with non-STEMI, PTX3 and B-natriuretic peptide (BNP) are independent predictors for cardiovascular events. ${ }^{23}$ In addition, PTX3 is involved in the pathogenesis of vascular damage. ${ }^{19}$ Norata $e t a l^{24}$ showed that PTX3 was a protective factor in patients with acute MI, especially in the reperfusion phase.

As an acute phase protein, CRP induces the expression of endothelial cell adhesion molecule-1, vascular cell adhesion molecule- 1 and monocyte chemoattractant protein-1. ${ }^{25}$ CRP is closely associated with the severity of atherosclerosis, ${ }^{3}$ which was in accordance with this study. The structure of PTX3 is similar to that of CRP. ${ }^{26}$ However, while CRP is mainly produced in the liver and can be induced by several non-specific diseases, PTX3 is produced in the cardiac circulation. ${ }^{8}$ On the other

Table 3 Characteristics of patients with renal dysfunction according to the Gensini scores

\begin{tabular}{|c|c|c|c|c|}
\hline & \multicolumn{4}{|c|}{ Gensini score } \\
\hline & $0-45$ & $46-90$ & $>90$ & p Value \\
\hline Cases (male) & $2(0)$ & $8(7)$ & $11(8)$ & \\
\hline Age (mean $\pm S D)$ & $71.5 \pm 6.4$ & $68.3 \pm 13.2$ & $75.7 \pm 12.4$ & 0.45 \\
\hline Weight (mean+SD) & $70.5 \pm 7.7$ & $60.9 \pm 6.6$ & $63.9 \pm 10.9$ & 0.42 \\
\hline Hypertension & 2 & 6 & 8 & 0.70 \\
\hline Diabetes & 0 & 1 & 4 & 0.34 \\
\hline HDL-cholesterol (mmol/L) & $1.6 \pm 0.6$ & $1.6 \pm 0.5$ & $1.4 \pm 0.3$ & 0.72 \\
\hline LDL-cholesterol (mmol/L) & $2.8 \pm 0.4$ & $2.8 \pm 0.8$ & $2.5 \pm 0.5$ & 0.94 \\
\hline Creatinine $(\mu \mathrm{mol} / \mathrm{L})$ & $113.5 \pm 2.1$ & $108.5 \pm 36.0$ & $122.82 \pm 46.2$ & 0.76 \\
\hline eGFR $\left(\mathrm{mL} / \mathrm{min} / 1.73 \mathrm{~m}^{2}\right)$ & $44.5 \pm 9.8$ & $50.2 \pm 8.1$ & $42.8 \pm 15.1$ & 0.46 \\
\hline PTX3 & $5.2 \pm 1.4$ & $7.1 \pm 1.2$ & $8.1 \pm 2.0$ & 0.09 \\
\hline CRP & $7.0 \pm 1.4$ & $18.5 \pm 27.4$ & $17.1 \pm 11.1$ & 0.74 \\
\hline
\end{tabular}


hand, PTX3 has a relatively long half-life and is more easily detected than CRP. In addition, PTX3 is less influenced by total cholesterol, high-density lipoprotein, haemoglobin, smoking, obesity or gender. ${ }^{27}$

The results of this study showed that PTX3 was highly expressed with increasing seriousness of atherosclerotic cardiac artery occlusion. In a study by Chen et al, ${ }^{13}$ PTX3 expression increased significantly in mice with acute renal damage. However, the association between PTX3 or CRP and patients with decreased eGFR is still unclear. In this study, we further investigated the association between the pentraxin superfamily and the severity of coronary artery stenosis in patients with CKD. Results showed that PTX3 was associated with the Gensini score in patients with decreased eGFR, while no such association was found for CRP, suggesting that PTX3 is correlated with the severity of coronary artery stenosis in patients with CKD, and that PTX3 could be a better predictor for the progression of coronary arterial lesions than CRP in patients with CKD. These results are in agreement with the study of Kanbay et al. ${ }^{14}$

This study is not without limitations. First, it was a cross-sectional study, and no follow-up analysis was performed. Second, the sample size was relatively small; this meant that the subgroups were even smaller, so the co-founders for multivariate analysis would be numerous. The statistical analysis should therefore be repeated in larger studies. In addition, further investigations could also be performed to evaluate the value of PTX3 in the risk evaluation of patients with CHD with renal dysfunction, as well as the predictive value of PTX3 for cardiorenal syndrome. We investigated PTX3 as a marker of severity of CHD but, importantly for prognosis, PTX3 has shown potential as a specific indicator for coronary plaque vulnerability; ${ }^{11}$ unfortunately, we did not have CT images for all of the patients in this study, so future studies should investigate the association of PTX3 and plaque instability in patients with CHD and renal dysfunction.

In conclusion, in this small-scale study, PTX3 was correlated with the Gensini score. In patients with CKD, PTX3 but not CRP was associated with the Gensini score, suggesting that PTX3 could be a more valuable predictor for the risk of CHD in patients with renal dysfunction than CRP. We intend to undertake further studies in larger populations to investigate whether PTX3 is as valuable as BNP in clinical practice; this will involve more detailed examinations including CT images.

Contributors $H L, S G$ and $X Q$ have made substantial contributions to conception and design; HL, SG, WF, FY and MZ have made substantial contributions to the acquisition of data, or analysis and interpretation of data; $\mathrm{HL}, \mathrm{SG}$ and $X Q$ have been involved in the drafting of the manuscript or its critical revision for important intellectual content; all authors have given final approval of the version to be published.

Funding This work was supported by the National Natural Science Foundation of China (81370361) and the science and technology commission of shanghai municipality Qu X (grant number 12140902800).
Competing interests None.

Patient consent Obtained.

Ethics approval Shanghai Chest Hospital.

Provenance and peer review Not commissioned; externally peer reviewed.

Data sharing statement Original data are available from the corresponding author, $X Q$, at Shanghai Chest Hospital, Affiliated to Shanghai Jiao Tong University, Shanghai 200240, China: qxkmedsci@163.com.

Open Access This is an Open Access article distributed in accordance with the Creative Commons Attribution Non Commercial (CC BY-NC 4.0) license, which permits others to distribute, remix, adapt, build upon this work noncommercially, and license their derivative works on different terms, provided the original work is properly cited and the use is non-commercial. See: http:// creativecommons.org/licenses/by-nc/4.0/

\section{REFERENCES}

1. Vucevic D, Radak D, Radosavljevic T, et al. [Inflammatory process in atherogenesis: new facts about old flame]. Med Pregl 2012;65:388-95.

2. Chironi G. [New biomarkers for cardiovascular risk evaluation]. Rev Prat 2012;62:783-5.

3. Maekawa Y, Nagai T, Anzai A. Pentraxins: CRP and PTX3 and cardiovascular disease. Inflamm Allergy Drug Targets 2011;10:229-35.

4. Correale M, Totaro A, Abruzzese S, et al. Acute phase proteins in acute coronary syndrome: an up-to-date. Cardiovasc Hematol Agents Med Chem 2012;10:352-61.

5. Kravitz MS, Pitashny M, Shoenfeld Y. Protective moleculesC-reactive protein (CRP), serum amyloid $P(S A P)$, pentraxin3 (PTX3), mannose-binding lectin (MBL), and apolipoprotein A1 (Apo A1), and their autoantibodies: prevalence and clinical significance in autoimmunity. J Clin Immunol 2005;25:582-91.

6. Braunwald E. Creating controversy where none exists: the important role of C-reactive protein in the CARE, AFCAPS/TexCAPS, PROVE IT, REVERSAL, A to Z, JUPITER, HEART PROTECTION, and ASCOT trials. Eur Heart J 2012;33:430-2.

7. Yousuf $\mathrm{O}$, Mohanty BD, Martin SS, et al. High-sensitivity C-reactive protein and cardiovascular disease: a resolute belief or an elusive link? J Am Coll Cardiol 2013;62:397-408.

8. Nebuloni M, Pasqualini F, Zerbi P, et al. PTX3 expression in the heart tissues of patients with myocardial infarction and infectious myocarditis. Cardiovasc Pathol 2011;20:e27-35.

9. Matsui S, Ishii J, Kitagawa F, et al. Pentraxin 3 in unstable angina and non-ST-segment elevation myocardial infarction. Atherosclerosis 2010;210:220-5.

10. Garlanda C, Bottazzi B, Moalli F, et al. Pentraxins and atherosclerosis: the role of PTX3. Curr Pharm Des 2011;17:38-46.

11. Soeki T, Niki T, Kusunose K, et al. Elevated concentrations of pentraxin 3 are associated with coronary plaque vulnerability. J Cardiol 2011;58:151-7.

12. Karakas MF, Buyukkaya E, Kurt M, et al. Serum pentraxin 3 levels are associated with the complexity and severity of coronary artery disease in patients with stable angina pectoris. J Investig Med 2013;61:278-85.

13. Chen J, Matzuk MM, Zhou XJ, et al. Endothelial pentraxin 3 contributes to murine ischemic acute kidney injury. Kidney Int 2012;82:1195-207.

14. Kanbay M, Ikizek $M$, Solak $Y$, et al. Uric acid and pentraxin-3 levels are independently associated with coronary artery disease risk in patients with stage 2 and 3 kidney disease. Am J Nephrol 2011;33:325-31.

15. Ringqvist I, Fisher LD, Mock M, et al. Prognostic value of angiographic indices of coronary artery disease from the Coronary Artery Surgery Study (CASS). J Clin Invest 1983;71:1854-66.

16. Willeke F, Assad A, Findeisen $\mathrm{P}$, et al. Overexpression of a member of the pentraxin family (PTX3) in human soft tissue liposarcoma. Eur J Cancer 2006;42:2639-46.

17. Gensini GG. A more meaningful scoring system for determining the severity of coronary heart disease. Am J Cardiol 1983; $51: 606$.

18. Ma YC, Zuo L, Chen JH, et al. Modified glomerular filtration rate estimating equation for Chinese patients with chronic kidney disease. J Am Soc Nephrol 2006;17:2937-44. 
19. Savchenko A, Imamura M, Ohashi R, et al. Expression of pentraxin 3 (PTX3) in human atherosclerotic lesions. J Pathol 2008;215:48-55.

20. Latini R, Gullestad L, Masson S, et al. Pentraxin-3 in chronic heart failure: the CORONA and GISSI-HF trials. Eur J Heart Fail 2012;14:992-9.

21. Kotooka N, Inoue T, Fujimatsu D, et al. Pentraxin3 is a novel marker for stent-induced inflammation and neointimal thickening. Atherosclerosis 2008;197:368-74.

22. Latini R, Maggioni AP, Peri G, et al. Prognostic significance of the long pentraxin PTX3 in acute myocardial infarction. Circulation 2004;110:2349-54.

23. Brugger-Andersen T, Ponitz V, Kontny $\mathrm{F}$, et al. The long pentraxin 3 (PTX3): a novel prognostic inflammatory marker for mortality in acute chest pain. Thromb Haemost 2009;102:555-63.
24. Norata GD, Marchesi P, Pulakazhi Venu VK, et al. Deficiency of the long pentraxin PTX3 promotes vascular inflammation and atherosclerosis. Circulation 2009;120:699-708.

25. Pasceri V, Willerson JT, Yeh ET. Direct proinflammatory effect of C-reactive protein on human endothelial cells. Circulation 2000;102:2165-8.

26. Bottazzi B, Vouret-Craviari V, Bastone A, et al. Multimer formation and ligand recognition by the long pentraxin PTX3. Similarities and differences with the short pentraxins C-reactive protein and serum amyloid P component. J Biol Chem 1997;272:32817-23.

27. Witasp A, Carrero JJ, Michaelsson K, et al. Inflammatory biomarker pentraxin 3 (PTX3) in relation to obesity, body fat depots and weight loss. Obesity (Silver Spring) 2014;22:1373-9. 